\title{
Quaderni
}

QUADERNI Communication, technologies, pouvoir

97 | Automne 2018

Néo-libéralisme(s). Réseaux et formes des

mobilisations en France

\section{Une participation politique renouvelée}

L'invention de modes individualisés et critiques d'engagement numérique en campagne électorale

Renewing political participation. The invention of individualized and critical modes of digital engagement in the election campaigns.

\section{Fabienne Greffet et Stéphanie Wojcik}

\section{OpenEdition}

Journals

Édition électronique

URL : http://journals.openedition.org/quaderni/1325

DOI : 10.4000/quaderni.1325

ISSN : 2105-2956

Éditeur

Les éditions de la Maison des sciences de l'Homme

Édition imprimée

Date de publication : 5 octobre 2018

Pagination : 107-131

Référence électronique

Fabienne Greffet et Stéphanie Wojcik, « Une participation politique renouvelée », Quaderni [En ligne],

97 | Automne 2018, mis en ligne le 05 octobre 2020, consulté le 04 janvier 2021. URL : http://

journals.openedition.org/quaderni/1325; DOI : https://doi.org/10.4000/quaderni.1325 


\section{Politique}

\section{Une participation politique renouvelée L'invention de \\ Depuis la fin des années 2000, les sites web dédiés et la plupart des réseaux sociaux sont appropriés par les candidats aux élections comme supports de diffusion d'informations, de communication et de mobilisation ${ }^{1}$. Cette situation concerne la majorité des pays européens ${ }^{2}$, et complète l'usage de plus en plus fréquent des traces numériques afin de tenter de mieux cerner les attentes des publics ou tester les messages de campagne ${ }^{3}$.}

\section{modes individualisés}

et critiques

d'engagement numérique en

campagne

électorale

Fabienne

Greffet

IRENEE (Nancy) et Pacte (Grenoble)

Université de Lorraine

Stéphanie Wojcik

CEDITEC

Université Paris Est Créteil
En ce qui concerne plus spécifiquement la France, les campagnes présidentielles de 2012 et 2017 présentent la particularité d'avoir été précédées de longues séquences pré-électorales puisque des « primaires ouvertes » ont été organisées successivement, en 2012, pour la désignation du candidat du Parti Socialiste et du Parti radical de gauche ; puis, en 2017, pour la désignation des candidats des partis écologistes et socialiste et de celui des Républicains ${ }^{4}$. Ces temporalités très étendues de campagnes ont été l'occasion pour les candidats et leurs équipes de multiplier les supports de communication web en vue de favoriser la mobilisation à la fois en ligne et hors ligne, y compris lorsqu'il s'agissait de mobiliser en dehors des primaires et des structures partisanes établies. En témoignent la mise en ligne, plus d'un an avant l'élection, de plateformes destinées à soutenir les (futures) candidatures d'Emmanuel Macron et de Jean-Luc Mélenchon ${ }^{5}$.

Ainsi, tout en étant très présents sur les réseaux socionumériques comme Facebook ou Twitter, les candidats et les partis n'ont pas délaissé les sites Internet ${ }^{6}$, pratiquant des webcampagnes « mixtes », qui associent des dispositifs relevant $\mathrm{du}$ « web $1.0 »$ et du « web 2.0 ». Celles-ci s'inscrivent également dans des contextes d'usage 
quasi généralisé d'Internet par la population. Selon les statistiques d'Eurostat et du CREDOC, $80 \%$ des foyers français disposaient d'une connexion Internet et 52\% des internautes de 12 ans et plus déclaraient qu'ils étaient inscrits sur au moins un réseau socionumérique en 2012. Ces proportions s'établissent respectivement à $86 \%$ des foyers et $65 \%$ des internautes en $2017^{7}$.

L'appropriation par les citoyens de ces dispositifs reste assez peu explorée, en dépit d'une multiplication des usages politiques des technologies numériques qui contribue à renouveler et à discuter la notion de participation politique ${ }^{8}$, et dans le cadre spécifique des campagnes électorales, celles d'engagement et de militantisme. En effet, si la majorité des activités permises par le web 1.0 peut largement entrer dans le paradigme traditionnel de la participation politique ${ }^{9}$, ce n'est pas le cas pour celles qui s'appuient sur le web 2.0 et les plateformes de médias sociaux qui y sont associées, même si la sociologie des utilisateurs reste très marquée par les inégalités bien connues dans la participation politique hors ligne ${ }^{10}$. Ainsi, se trouve ravivée la controverse entre des auteurs qui considèrent que le web recèle des formes de participation politique inédites ${ }^{11}$ et d'autres qui estiment au contraire que se déploient sur le web des formes « faibles » d'engagement, qui seraient même nuisibles à l'action politique sur le terrain supposée plus efficace ${ }^{12}$.

Cette contribution analyse la question de la participation et des formes d'engagement politiques des internautes à travers des entretiens réalisés auprès d'internautes actifs durant une campagne présidentielle française. Dans le cadre du projet enpolitique.com ${ }^{13}$, nous avons en effet souhaité dégager la participation politique en ligne de ces perspectives normatives, conduisant généralement à rechercher la mise en équivalence d'indicateurs élaborés pour les situations hors ligne avec des pratiques en ligne ${ }^{14}$. À la suite notamment de Bennett et Segerberg ${ }^{15}$ ou Bimber $^{16}$, nous nous situons ici dans la perspective selon laquelle de nouvelles formes de participation émergent, en raison des appropriations croissantes des dispositifs communicationnels fondés sur Internet et le web. Ces formes de participation conduisent notamment à repérer des actes participatifs numériques plus ou moins reliés aux campagnes électorales. Notre propos consiste à mettre en lumière deux aspects de ces formes de participation : d'une part, celui de la créativité des internautes, à travers la tension entre appropriations du numérique en campagne électorale et « management », voire directivité de la part des équipes qui promeuvent les candidats ${ }^{17}$; d'autre part, celui de la tension entre une participation qui repose sur la mobilisation de leurs réseaux sociaux, et la prise de risque que cela représente pour les internautes. Au préalable, nous présentons une typologie des actes participatifs en ligne qui sert de point d'appui à l'exploration de ces deux dynamiques.

\section{Une typologie des actes participatifs en ligne}

L'opérationnalisation de la notion de participation politique en ligne a conduit ces dernières années à plusieurs propositions de classifications reposant sur une grande variété d'indicateurs. À titre d'exemples, Bakker et de Vreese distinguent des formes de participation politique actives et passives en ligne ${ }^{18}$. D'autres auteurs tels que Cantijoch et alii décrivent trois types d'activités 
politiques possibles en ligne : le type «E-news » correspond à la recherche d'informations, considérée comme «passive » (par exemple, le fait de visiter un site de campagne ou de regarder des vidéos en relation avec celle-ci) ; le type « E-party » implique des activités numériques orientées vers les organisations, avec notamment l'inscription sur les sites de partis, l'utilisation des outils de mobilisation fournis par les partis, la création ou l'adhésion à un groupe politique sur Facebook; le type « E-expressive » concerne plus particulièrement la « participation expressive », notamment le fait de poster, partager ou intégrer sur un blog des contenus politiques ${ }^{19}$.

Ces catégorisations ne semblent pas complètement satisfaisantes, notamment en ce qui concerne la distinction entre formes « actives » et « passives » de participation politique sur le web : le fait de s'inscrire à un groupe Facebook politique, par exemple, ne suppose pas forcément d'être plus « actif» que de chercher de l'information sur un site de candidat.

C'est pourquoi nous avons proposé une nouvelle typologie qui nous semble permettre une analyse plus fine des pratiques de participation politique en ligne en contexte électoral. Cette typologie, élaborée en collaboration avec Gersende Blanchard, comprend six catégories d' « actes participatifs en ligne $»^{20}$ dont nous proposons ci-dessous une représentation simplifiée, ainsi qu'un descriptif sommaire.

\section{Graphique : typologie des « actes participatifs » en ligne}

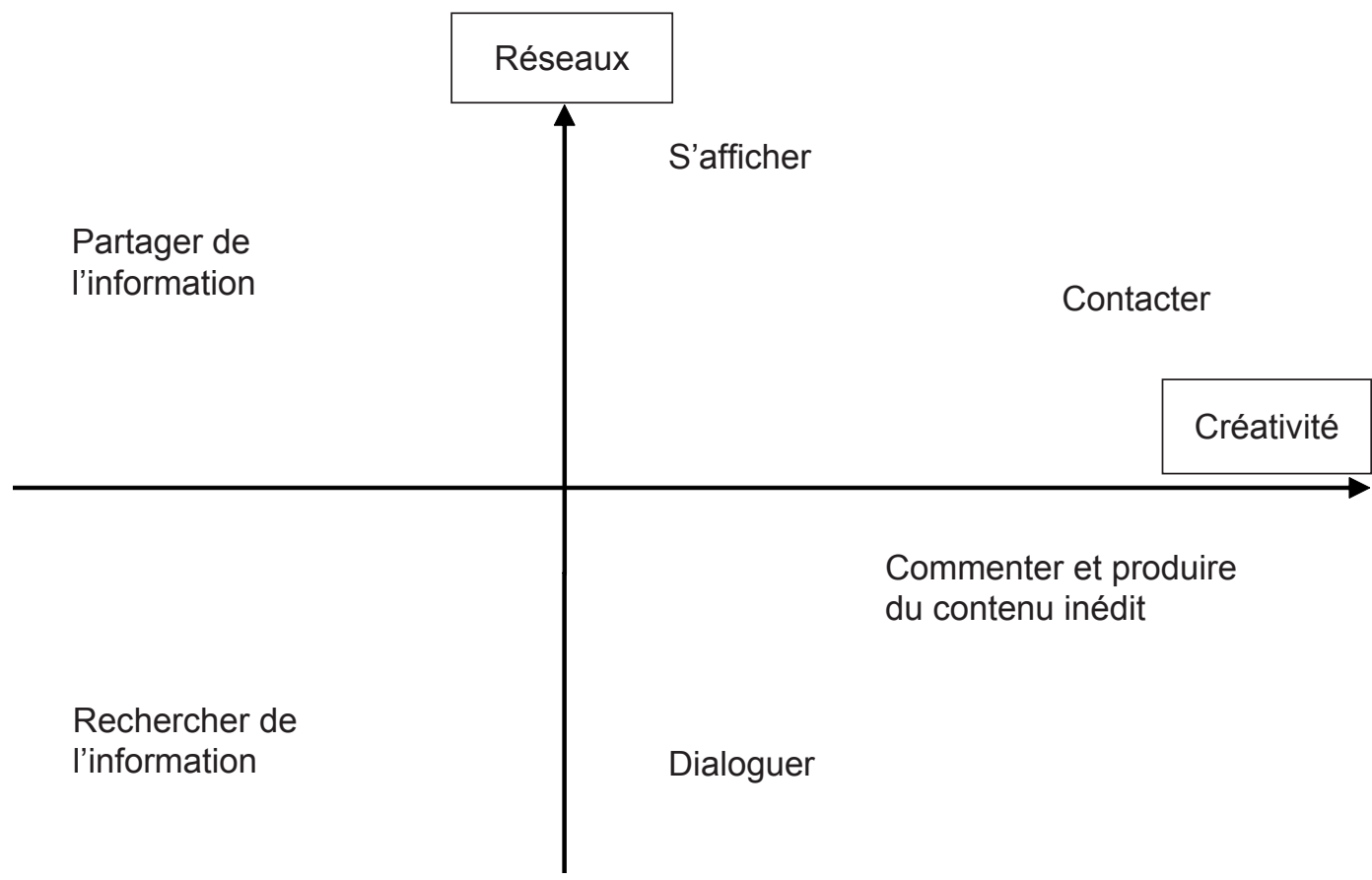


Deux critères - représentés en abscisse et ordonnée du graphique - sous-tendent l'élaboration de cette typologie. Le premier est celui du degré de créativité offert à l'utilisateur, un élément généralement ignoré dans les classifications, surtout lorsqu'elles sont enchâssées dans des conceptions de la participation politique antérieures à la diffusion des supports numériques de communication. La créativité, ou capacité à agencer soi-même des contenus, dans la cadre d'un dispositif particulier (et porteur de contraintes) constitue en effet une dimension centrale de la " culture numérique », expression le plus souvent employée pour désigner un ensemble de caractéristiques propres aux pratiques culturelles médiées par le web ${ }^{21}$ et qui concernerait notamment les jeunes générations. Pour Jenkins et al., chez les « natifs digitaux », les pratiques culturelles seraient de plus en plus poreuses avec l'engagement politique ${ }^{22}$. C'est pourquoi cette dimension a été prise en compte dans différents indicateurs visant à mesurer la participation politique en ligne durant la campagne présidentielle de 2012. Par exemple, nous demandions aux internautes s'ils avaient publié un billet de blog, laissé un commentaire sur une page de candidat ou de parti, « live-twitté » ou publié un statut Facebook en relation avec la campagne. Ces différents indicateurs abondent la catégorie d'actes participatifs consistant à « commenter et produire du contenu inédit ».

Un deuxième critère est celui de la présence d'une participation politique « en réseau » (digitally networked participation), définie par Theocharis comme : « une action personnalisée, basée sur des médias en réseau, qui est menée par des citoyens individuels avec l'intention de déployer leur propre mobilisation et d'activer leurs propres réseaux sociaux $(\ldots) »^{23}$. Nous distinguons donc des formes de participation politique « en réseau », qui mettent en jeu le réseau social de l'internaute. À titre d'exemples, le fait de rejoindre un groupe Facebook défendant ou attaquant un parti politique ou un candidat, d'ajouter une image ou une bannière partisane à son profil ou son journal Facebook, de « liker » le statut d'un ami, d'un parti ou d'un candidat ou d'apposer un badge à l'effigie d'un candidat ou un slogan sur un compte Twitter constituent des formes de participation « en réseau » : sans produire du contenu, l'internaute met en scène son soutien à un candidat ou un parti politique. De la même façon, lorsqu'il s'agit de partager des informations, de « re-tweeter » un message concernant la campagne, ou de diffuser une blague, l'internaute implique son réseau relationnel dans son action de participation.

Nous avons, dans d'autres contributions, quantifié la pratique par les internautes de ces « actes participatifs » et les caractéristiques socio-politiques qui leur sont statistiquement le plus souvent associées, en utilisant les résultats d'un questionnaire en ligne, auto-administré à 827 répondants ${ }^{24}$. Dans cet article, nous analysons l'engagement des internautes durant cette campagne, à travers une approche plus qualitative des dimensions de notre typologie.

\section{Une approche qualitative de la participation politique en ligne}

Nous présupposons en effet que, pour mieux saisir le sens et la teneur des « actes participatifs » quantifiés, il importe de les saisir qualitativement, soit en procédant à des analyses de corpus - 
que nous n'effectuons pas ici -, soit à travers des entretiens semi-directifs qui proposent un espace de réflexivité sur leurs pratiques à des internautes impliqués dans les campagnes. Cela permet d'approcher autrement les dimensions de la participation politique en ligne. Ainsi, parmi les répondants à notre enquête par questionnaire précédemment évoquée, nous avons pu rencontrer vingt internautes, de différentes sensibilités partisanes grâce auxquels nous avons pu explorer plus finement les modalités et les représentations de leur engagement dans la campagne présidentielle.

Avec cette démarche, nous nous situons à rebours du développement de l'exploitation de grandes masses de données, issues de certains dispositifs du web $2.0^{25}$, au profil d'une d'enquête de sciences sociales qui conserve un intérêt certain pour la recherche, notamment pour évaluer comment les internautes s'approprient les dispositifs numériques pour faire campagne.

Nous approchons également la participation politique à partir d'une définition « compréhensive», au plus près des usagers, qui est la suivante : « n'importe quelle activité en ligne, individuelle ou collective, qui engage les personnes dans la politique ou la critique de la politique, que cela entraîne ou non des effets directs sur les affaires publiques $»^{26}$. Avec cette définition, il s'agit d'envisager des formes d'action qui, contrairement à la définition canonique de Verba et Nie, n'ont pas nécessairement une dimension de mise en cause directe des autorités publiques ; mais aussi d'introduire les notions d'interpellation et d'engagement, qui suppose donc que les usagers confèrent un sens politique à leurs pratiques numériques, même si celui-ci peut être porteur d'ambiguïtés. Cette définition ne distingue pas entre participation, engagement et militantisme, qui apparaissent particulièrement enchevêtrés dans les discours des enquêtés.

Plus précisément, le guide d'entretien s'articule autour de trois thématiques : une thématique sur les pratiques d'Internet de l'internaute pendant la campagne, à partir d'une consigne ouverte et qui ne faisait pas explicitement référence aux technologies numériques («J'aimerais que vous disiez ce qui vous a marqué pendant la dernière campagne présidentielle ») ; une thématique sur les perceptions des campagnes en ligne, amenant notamment l'internaute à s'exprimer sur ce qu'ils pensaient de ce que les candidats et partis proposaient en ligne durant la campagne ; et une troisième thématique, de réaction à trois matériaux de campagne (deux vidéos et un jeu en ligne). Les entretiens ont duré entre une heure et deux heures trente. Ils ont été réalisés à distance de la campagne elle-même, entre la fin juin 2012 et janvier 2013, avec une forte concentration sur la période octobre-novembre 2012 (17 entretiens sur 20).

L'enquête révèle une population sociologiquement assez typée, plus masculine, plus diplômée et plus politisée que la moyenne des Français : ce ne sont donc pas des profanes que nous avons rencontrés mais des individus qui ont souvent pris part à la campagne sur le terrain, relativisant d'emblée l'idée selon laquelle la présence en ligne entraînerait le désengagement des situations de face-à-face.

Cette population internaute, sympathisante ou déjà engagée, est celle qui est ciblée par les 
équipes de campagne en vue de susciter et mettre en scène la mobilisation du public. Dans les campagnes électorales des dernières décennies, en particulier aux États-Unis, il est en effet bien documenté que les directions de campagne tentent de mettre à profit les technologies numériques en vue non pas de dialoguer ou de construire un programme en partie avec l'aide des internautes, mais de mobiliser, de contrôler, de diriger l'activisme des militants, sympathisants et électeurs de manière à renseigner des objectifs précisément définis. Dès 2006, P. Howard évoquait ainsi des « citoyens managés », perspective ensuite approfondie à plusieurs reprises par Daniel Kreiss ${ }^{27}$. À la collecte numérique ou manuelle de données sur les électeurs, s'ajoutent des logiques de récolte de fonds, surveillance et management du volontariat (management volunteerism) ${ }^{28}$, partiellement automatisées et fondées sur l'utilisation stratégique d'algorithmes. La campagne présidentielle française de 2012 n'échappe pas à ces logiques, les équipes des candidats s'adjoignant le concours massif de logiciels de mobilisation électorale destinés notamment à transmuter les sympathies virtuelles en engagements sur le terrain et dans les urnes ${ }^{29}$. Comment les internautes réagissentils à cette offre de mobilisation électorale ? Se coulent-ils facilement dans le moule des options numériques déterminées par les partis et les candidats ? Ces derniers peuvent-ils réellement « manager» l'activisme numérique ? Nous faisons l'hypothèse que les consignes diffusées et les dispositifs mis en œuvre par les équipes leur échappent partiellement au profit de modes individualisés du faire-campagne, renforcées par les potentialités de créativité propre à la participation numérique. Cette créativité dans l'invention de soi, entendue « non pas comme une marque d'autonomie de l'individu mais plutôt comme une manière de "faire avec» des éléments fournis $»^{30}$ est travaillée en ligne comme hors ligne par les enjeux de l'interaction, notamment l'impératif goffmanien de construire sa face, auquel l'internaute se confronte lorsque sa participation politique en ligne met en jeu son réseau relationnel.

\section{L'information, préalable à la participation politique en ligne}

Avant d'analyser plus avant les actions de campagne, rappelons que la participation politique en ligne est toujours sous-tendue par des pratiques d'information ${ }^{31}$. Celles-ci sont les modes d'utilisation d'Internet les plus répandus et les plus fréquents, y compris durant les campagnes électorales ${ }^{32}$. Ce sont également les pratiques les plus souvent citées par les personnes rencontrées, lorsqu'on leur demande ce qu'elles ont fait sur Internet durant la campagne. Elles renvoient spontanément et sans hiérarchiser à une pluralité de sources : sites de médias traditionnels français et étrangers, pure players, blogs, de journalistes ou de militants, réseaux socionumériques... Comme concernant la participation politique hors ligne, ceux qui participent et s'engagent sont donc ceux qui s'exposent le plus à l'information. Et, comme en matière de pratiques d'information hors périodes de campagne, les personnes qui s'informent de façon extensive disposent également de suffisamment de ressources intellectuelles pour recouper et sélectionner l'information. Ces personnes produisent un discours critique sur les sources qu'elles mobilisent : « la propension à diversifier les sources pour comparer les faits et leur traitement, à approfondir les informations, à consulter l'expertise critique des spécialistes, ou 
encore à se sentir habilité pour prendre la parole, est l'apanage des lecteurs les plus diplômés, et tout particulièrement des professions à composante intellectuelle $»^{33}$.

On en trouve une illustration ci-dessous, marquée par le positionnement politique de l'enquêtée, de la part d'une adhérente UMP :

« Alors, je suis quelqu'un qui lit énormément, et la presse française, et la presse, la presse étrangère qui est un peu plus juste que la presse française, parce que vous savez quand même qu'elle est pro-gauche hein, malheureusement, hein.... Et pas très neutre et c'est ce que je reproche, hein, à l'heure actuelle, dans cette campagne d'ailleurs, ça a fait beaucoup de tort. Moi j'aimerais bien qu'on revienne à des journalistes qui soient neutres et qui, qui fassent leur travail de journalistes, non pas prennent position pour l'un ou pour l'autre. Parce que si on veut aider les gens, c'est pas en leur imposant nos idées, c'est en essayant de faire sortir les leurs, que c'est intéressant. Et... donc je vais beaucoup sur les sites étrangers, les sites canadiens, les sites israéliens, y a un très bon site en Israël où ils sont extrêmement percutants. ${ }^{34}$

De ce point de vue, les enquêtés sont plus proches du profil des personnes les plus politisées que de l'ensemble des consommateurs de médias numériques, statistiquement plutôt jeunes (moins de 34 ans), souvent étudiants ou inactifs, et présentant un rapport distant à la politique (faible intérêt pour la politique, peu de conversations politiques, tendance à ne se classer ni à gauche ni à droite $)^{35}$.

Il n'est pas rare que les pratiques d'information de ces enquêtés politisés et plus âgés passent par Twitter, notamment pour les enquêtés qui travaillent dans le secteur informatique, comme cet informaticien non encarté mais qui se situe à gauche :

«... Oui, bon, Libé, enfin le triptyque usuel, Libé, Le Monde, Figaro, même si bon politiquement parlant j'aurais plutôt tendance à me concentrer sur d'abord Le Monde et après, Libé et de temps en temps Le Figaro. Oui, soyons complètement honnête, il est assez rare que je me balade sur la page de garde du Figaro, quoi, c'est plus des liens qui me sont envoyés par Twitter qui font que... ${ }^{36}$ Les sources des réseaux socionumériques s'ajoutent en fait aux sources habituelles, et induisent une accélération du rapport à l'actualité :

«J'ai, depuis le mois de janvier 2012 jusqu'au mois de juin 2012, j'ai énormément été sur Twitter. Donc, j'ai vécu... une expérience intéressante, pour quelqu'un comme moi qui suis plutôt bien informé, qui lit la presse, qui regarde les émissions de télévision, etc., c'est-à-dire c'est l'actualité avant l'actualité. Donc, ça veut dire qu'on était, on avait l'impression, dans la journée, en suivant sur Twitter une information, que $20 \mathrm{~h}$ du soir, c'était déjà les informations, que c'était déjà un livre, un, que c'était déjà, comment dire, un reportage historique ou une émission historique. ${ }^{37}$

Parmi les sources d'information fréquemment mentionnées figurent des supports produits par les organisations de campagne : la possibilité de visionner les meetings, soit en direct, soit par des vidéos différé ${ }^{38}$. Ils constituent, notamment 
pour les adhérents et sympathisants de droite, qui sont nombreux à regarder Nicolas Sarkozy, une source perçue comme un complément indispensable, beaucoup plus complet et plus adéquat à leur sensibilité à la campagne que la télévision :

«Entre les deux tours, on n'a pas eu de meetings retranscrits donc sur Internet, fallait aller voir. Donc, ça c'était important. Si on se contentait de la télé, on n'avait rien. On n'a eu qu'un débat. Le fait de pouvoir aller voir les meetings sur Internet, c'était quelque chose d'important, sinon on en était privé. ${ }^{39}$

Visionner les meetings peut aussi, pour les militants les plus actifs, constituer un substitut, un moyen de rester impliqué dans la campagne même lorsqu'il n'est pas possible d'assister aux meetings, ce qui est le cas pour cet enquêté sans emploi, en difficulté financière au moment de la campagne :

«Donc j'étais pas très bien non plus... Dans ma tête je me... J'étais pas très... Et puis financièrement aussi... C'était difficile aussi de se déplacer même si... Et comme j'étais pas adhérent de l'UMP, j'étais pas au courant de tout... Je suivais tous les meetings en direct, en live, via Dailymotion. Tous, tous, je l'ai tous vu. Même Hollande, j'en ai suivi, même si ça m'a fait saigner les oreilles, mais je les ai regardés! Le Pen aussi. Très honnêtement, le seul que j'ai pas pu suivre, c'était Mélenchon. ${ }^{40}$

Plébiscité, le meeting apparaît comme une source « brute », peu travaillée par les enjeux propres à l'organisation : sa scénographie n'est jamais interrogée par les interviewés. En revanche, lorsque l'information dispensée par le parti ou l'organisation de campagne apparaît comme factice ou travaillée par les enjeux internes à l'organisation, elle est rejetée :

« Et je n'ai pas coché la case «j'accepte de recevoir des mails du parti socialiste» parce que je ne... je prête aucun crédit à la communication institutionnelle qu'elle vienne des entreprises, des partis politiques, de l'État.» ${ }^{41}$

Mais, ce qui intéresse le plus les personnes engagées en ligne, c'est de lire des personnes qui leur ressemblent. Les informations en provenance d'autres militants sont donc souvent mentionnées comme une source précieuse et précise :

«Il y a des débats à suivre entre partisans, qui sont un véritable régal intellectuel et visuel, on voit les arguments qu'ils s'échangent les uns les autres, et en fait, c'est l'occasion de sentir avec un parti, de dire : " oui effectivement il dit ça, je suis d'accord avec lui, il le formule de manière plus pertinente que moi j'aurais pu le formuler, ou au contraire pas dans le sens là, attaque plutôt sur ce point là ", et ça c'est quelque chose de complètement nouveau, en fait, les forces en présence dans une bataille politique, sont sur un terrain où elles peuvent se voir, on est passé de l'époque du sniper par alliés interposés à un clash quoi. » ${ }^{42}$

Cette source est aussi perçue comme étant plus complète et surtout plus fouillée que l'information disponible sur les comptes officiels de campagne.

«Ben, tout ce qui est information officielle, institutionnelle des partis, ça ne m'intéresse pas sur 
Internet. (...) Ce qui m'intéresse plus, c'est, un peu justement, les blogs ou les trucs un peu... les points de vue aussi des militants, etc. ${ }^{43}$

Ou, comme le dit un autre enquêté, ancien communiste, il s'agit d'éviter des sources officielles qui «formatent» les points de vue et tendent à traiter les enjeux politiques de façon superficielle :

" Parce qu'il y a le contenu de la campagne et y a les outils de diffusion et de propagande. Y a un effet cumulatif, c'est-à-dire que la façon dont ils ont ... ils ont formaté leur campagne... les tirait justement à éviter d'aller au fond des choses. » ${ }^{44}$

\section{L'environnement contraint de la participation aux campagnes officielles}

Cette opposition entre des sources riches, émanant généralement de militants, et les discours officiels des partis et des candidats se double d'une critique récurrente, par les interviewés, des contraintes qui encadrent l'ensemble des actions de mobilisation et des obstacles à l'interaction avec les acteurs politiques durant la campagne. Les discours des internautes laissent percevoir, plutôt qu'une créativité ou une satisfaction de s'exprimer, une gêne face à des dispositifs qui les conduisent à se conformer à des façons de faire campagne imposées par les partis et organisations de campagne.

Ces discours s'inscrivent dans un mouvement plus général, partagé par une partie du monde académique, qui tend à souligner - et parfois dénoncer - la difficulté des institutions à s'approprier les technologies numériques. Dès la création de sites par les partis politiques, des recherches ont montré que ceux-ci ne constituaient que très rarement des espaces d'interaction et de dialogue avec les internautes ${ }^{45}$. Concernant les pratiques des réseaux socionumériques par les leaders politiques, Lilleker et Jackson parlent de « web 1.5 » pour désigner leur dimension peu interactive, dans un environnement où la participation des internautes est très contrôlée ${ }^{46}$. La difficulté de l'articulation entre mise en scène des candidats et organisations, et « mise en conversation de la politique $»^{47}$ est confirmée à bien des égards par les internautes que nous avons rencontrés. Les velléités d'interaction des enquêtés se trouvent entravées par le « manque de sincérité » et l'impersonnalisation des comptes dont les candidats disposent sur les réseaux Ainsi, un enquêté évoque l'absence d'échanges qui caractérise le compte Twitter du Parti socialiste et de son candidat, ce dernier émettant essentiellement des messages formatés :

«Et... pareil, ça manquait de... de sincérité par exemple, on voyait des comptes, le compte Twitter $d u$ PS c'était du message automatique. Y avait aucune sincérité qui reflétait. Valait mieux suivre, les comptes Twitter de militants avec lesquels on pouvait avoir un débat. On pouvait avoir une réaction. Et avec le compte Hollande, on n'avait pas de réactions. On pouvait envoyer des tweets, des tweets assez agressifs, des questions mais on avait peu de réponses. ${ }^{48}$

En effet, la nécessité de contrôler étroitement la parole du candidat s'accommode mal de la réactivité qui semble constitutive des attentes des publics et une injonction de la communication politique sur les réseaux sociaux, avec les risques 
de détournement que cela comporte. Aussi, si en théorie, les acteurs politiques pourraient sur Twitter valoriser des éléments de spontanéité et de proximité, ce qui est véritablement apprécié des internautes, leurs pratiques de ce dispositif en campagne n'apparaît pas comme le lieu d'un véritable renversement des modalités de relation et d'échange entre représentants et représentés ${ }^{49}$.

Dès lors, la professionnalisation croissante de la communication politique numérique et l'élaboration de stratégies de campagne sont évaluées de manière paradoxale par nos interviewés. D'une part, les tentatives d'appropriation par les acteurs politiques des dispositifs du web 2.0 font l'objet de critiques acerbes, comme l'illustrent ces propos d'une autre enquêtée, sympathisante du Front de Gauche, qui souligne le caractère artificiel, révélateur d'une démarche strictement stratégique des comptes Twitter des candidats de l'UMP et du PS :

« (...) je trouvais que c'était factice, enfin, un compte de Sarkozy ou de Hollande ou de, enfin, c'est tellement réfléchi derrière tout ça donc, le peu que je suivais c'était, voilà c'était plus des petites personnes, des personnalités qui étaient à côté, qui étaient peut-être plus à même d'avoir des réactions un peu, un peu plus personnelles, ou un peu plus, moins, peut-être moins dictées, ... $»^{50}$

D'autre part, certains enquêtés se félicitent d'une telle professionnalisation, entendue comme la préparation minutieuse de chaque événement qui rythme la campagne de leur candidat, et relèvent au contraire la volonté de celui-ci de les inclure dans sa stratégie. Ainsi, une enquêtée valide l'hypothèse élaborée en 2006 par Philip Howard sur le « management » des citoyens durant les campagnes électorales :

«Alors c'est vrai que les partis politiques ont très, très bien préparé la campagne. Puisqu'y avait quand même des diffusions, $y$ avait des retransmissions, y avait ... on est extrêmement associés à tout ce qui se passe, on est en permanence contacté, on est... vraiment, l'engouement continue (...) Et cette mise en place, cette façon d'englober tous les internautes continue, et les partis politiques sont vraiment à la recherche de gens comme nous, voilà. $1^{51}$

Cet encadrement du bénévolat, relevé à multiples reprises dans la littérature consacrée notamment aux campagnes américaines, s'accompagne de la mise en œuvre de dispositifs destinés à faire participer les sympathisants à l'organisation ou à la teneur de la campagne. Lors de cette campagne, les entretiens donnent à voir trois grands types d'initiatives par lesquelles les partis et les équipes de campagne entreprennent de mobiliser en ligne leurs sympathisants.

Il s'agit d'abord d'utiliser les fonctionnalités de certains dispositifs du web 2.0 pour prendre la mesure de la popularité d'une décision ou d'une proposition de campagne comme l'explique cette sympathisante UMP à propos de l'utilisation de Facebook :

«Alors, ça peut être sur des propositions de l'UMP, de Nicolas Sarkozy. Ou ça pouvait être les propositions des autres candidats puisqu'avant le premier tour on avait les autres. Et donc, on nous demandait de donner notre avis si oui ou non on trouvait que c'était bien ou pas et après 
on avait le pourcentage. $»^{52}$

S'apparentant à une sorte d'enquête d'opinion rapide, non représentative, ce type de mesure ne constitue pas un débat argumenté et contradictoire sur les propositions du candidat.

Ensuite, les partis et équipes de campagnes mettent en place des dispositifs beaucoup plus sophistiqués, destinés non pas à appréhender l'opinion des internautes, mais à valoriser leur engagement pour le candidat. De tels dispositifs, souvent conçus par des agences de communication, promeuvent une conception du militantisme qui se heurte souvent à celle défendue par ceux auxquels il s'adresse. Par exemple, le site de campagne du candidat du MoDem contenait une section intitulée « Les Volontaires » précisément dévolue à celles et ceux qui souhaitaient faire campagne pour François Bayrou, sans toutefois s'engager formellement dans son parti ou de manière trop explicite. Les internautes étaient ainsi incités à réaliser telle ou telle action (par exemple, diffuser une vidéo de François Bayrou sur leur page Facebook, inviter leurs famille et amis à assister à l'un de ses meetings...) qui leur permettait de créditer leur compte d'un certain nombre de points (appelés « décibels » ou « $\mathrm{dB} »)$ selon le principe de la gamification existant par ailleurs dans tout un ensemble d'autres applications, dispositifs et services du web (Foursquare...) ou campagnes menées par des entreprises privées. L'un des enquêtés reste sceptique vis-à-vis d'un tel dispositif :

" Parce que c'est compliqué, parce que déjà, il faut connaitre ce que ça veut dire $d B$. C'est compliqué, il faut... il faut... voir, enfin... C'est compliqué, en même temps, c'est infantilisant, c'est assez curieux. C'est-à-dire que, que... cette histoire là un petit peu de cumuler des points, comme si on était à la foire. Et puis... et en même temps, compliqué parce qu'y a différents degrés, alors pourquoi c'est... pourquoi on fait plus de bruit quand on invite des gens aux meetings de Lille, plutôt que d'aller coller une affiche? Enfin, je sais plus, y avait pas ça mais bon... C'est décidé par qui ? Pff, c'est compliqué (rires). Ça me laisse pantois. $\gg^{53}$

Un autre, adhérent Jeune Populaire, dénonce, à propos d'un dispositif assez analogue proposé par l'UMP, ce qu'il considère comme contraire à une logique militante qui serait celle du dévouement et de la solidarité collective :

« Je m'inscris pas dans cette logique, je pense. Parce que, c'est, voilà... c'est de la concurrence entre les militants. Je considère ça un peu comme une dérive, ça, en soit. Si on fait ça, si on fait de la politique, c'est pas pour... c'est pas pour gagner des cadeaux ou quoi que ce soit, voilà. $\rangle^{54}$

Enfin, les partis et équipes de campagne se prêtent volontiers à la mise en scène d'actions de communication dont sont particulièrement friands les médias mainstream, alors même qu'elles ne concernent qu'une minorité d'internautes. Par exemple, les sessions de live-tweets et de « riposte parties » à l'occasion d'un passage télévisé des candidats et du débat de l'entre-deuxtours donnent lieu à des scénographies, sensiblement identiques selon les partis et les candidats, où sont mises en scène des « communautés » de militants le plus souvent jeunes, enthousiastes et prompts à saisir en quelques mots les propos de 
leur candidat et à contrer efficacement et avec humour les attaques verbales de ses opposants. Pour certains enquêtés, la narration de telles sessions dans la presse donne une image trompeuse de l'engagement des militants, comme le décrit un adhérent PS attaché aux mobilisations sur le terrain :

«Ça et... Alors que, quoi, je... alors qu'on savait, enfin voilà, il $y$ avait vingt personnes qui avaient... vraiment été actives la veille et pourtant, on lisait les journaux le lendemain, on en discutait entre nous, on avait l'impression qu'il $y$ avait des milliers de personnes quoi. (...) Et... c'est vrai qu'on a... moi, depuis tout à l'heure là, $j$ 'ai en tête, vraiment... les photos que j'ai vues des war rooms de Hollande en fait, c'était une cinquantaine de personnes. Et... moi, voilà, quand on me pose la question "qu'est-ce que c'est la campagne d'Hollande? », j'ai ça en tête. Ces photos là. $\|^{55}$

L'encadrement de la communication politique fait l'objet de nombreuses critiques de la part des enquêtés, qu'il s'agisse pour eux de déplorer des interactions tronquées ou de se distancier des dispositifs offerts par les partis et les équipes des candidats. De telles tensions apparaissent dès lors que sont déployées au sein des organisations des techniques de campagne standardisées, légitimées par la croyance en leur " efficacité " et leur « scientificité ", et médiatisées. Ainsi, la systématisation du porte-à-porte est perçue comme inauthentique et contrevenant $« \grave{a}$ l'ethos de la gratuité militante ${ }^{56}$. Néanmoins, au contraire du porte-à-porte, qui se pratique collectivement et en relation relativement étroite avec l'organisation, les pratiques de participation promues dans la campagne numérique peuvent s'organiser de manière plus individuelle. De ce fait, les réticences des personnes engagées à s'approprier les dispositifs officiels peuvent trouver un débouché dans d'autres formes d'engagement numérique, particulièrement parmi les personnes sympathisantes ou ayant pris leurs distances avec l'organisation.

\section{Créer son espace, inventer sa campagne}

Plutôt que de s'inscrire dans les dispositifs proposés, les personnes que nous avons rencontrées tendent en effet à inventer leurs propres façons de se mobiliser et de mobiliser.

Une première façon de faire, que ce sont appropriée des adhérents de partis insérés dans les organisations, consiste à créer des pages locales de partis politiques sur les réseaux socionumériques, afin de promouvoir et soutenir des (et leurs) actions militantes. Ces pages peuvent avoir été créées en amont et se pérenniser après le scrutin. En revanche, elles s'animent davantage en période de campagne. La création de ces pages s'effectue dans et au nom de l'organisation et l'engagement en ligne s'inscrit alors dans une démarche collective.

Le caractère collectif et proche des organisations est bien décrit par un étudiant en médecine, adhérent communiste depuis le $1^{\text {er }}$ mai 2011, et qui s'est retrouvé rapidement co-administrateur de deux pages Facebook locales du parti, créées à l'automne 2011, comme il l'explique dans l'extrait suivant :

«-Le blog c'est pas moi qui l'anime, c'est un 
autre camarade qui s'en occupe, mais bon c'est un peu toute la section qui donne des informations, ... parce qu'on a une liste mail, mais en fait, c'est des gens qui envoient des textes dessus, qui peuvent être bien pour le blog avec des photos, etc. Et puis on a quelqu'un qui s'occupe de ça, qui met sur le blog et puis moi après je relaye ce qu'il y a sur le blog sur les réseaux sociaux, etc. Donc, ouais c'est...bah ça s'est organisé un peu tout seul comme ça. Mais pour l'instant ça va, c'est un peu, c'est pas mal je trouve (rires). - Et vous avez repris ça pendant la campagne ou avant ou...?

- Bah ça c'est mis en place vraiment pendant la campagne, pour moi ce que je dis la campagne c'est : elle commence un an avant le...la date de l'élection quoi. Alors, bah moi j'ai adhéré, j'ai adhéré donc le 1'r mai 2011. Et puis... Ça c'est mis en place, allez, à la rentrée quoi. Vers septembre, octobre.

- D'accord. Et là vous êtes devenu administrateur de la...enfin des deux pages?

- Bah sur les pages Facebook, on est plusieurs à avoir les mots de passe... qui veulent s'investir dessus et donc on est plusieurs à...voilà à s'occuper de ça.

- D'accord. Vous êtes combien à peu près par...? - On est... je crois qu'on est quatre. $»^{57}$

Un autre étudiant, UMP celui-là, décrit comment la page des Jeunes Populaires créée dans sa circonscription au cours de la campagne constitue un lieu de (modeste) rassemblement militant, d'une cinquantaine de personnes :

« - Donc on a créé une page pour les jeunes populaires, les jeunes de l'UMP de notre circonscription. Qui réunit à la fois, militants, sympathisants, adhérents. Et on débat à la fois de l'actualité nationale, locale, chacun donne son avis. Et on se sert à la fois de cette page pour créer des évènements, des réunions, pour rassembler un maximum de personnes.

- Et... vous l'avez créée quand, à peu près, cette page?

- Au mois de... au mois de mars. À partir du moment où la campagne a commencé à s'intensifier. (...)

- Et tout ces personnes ont plus ou moins participé à la campagne... de Nicolas Sarkozy ?

- Ouais. Chacun en fonction de ses moyens. Donc, il y en a qui ont... fait la démarche d'adhérer, de nous rejoindre, et d'autres qui ont milité avec plus... de modération j'ai envie de dire, mais... qui ont fait... au final le choix de Nicolas Sarkozy. $~^{58}$

La création et l'administration de ces pages, portée le plus souvent par des adhérents jeunes, familiers des technologies numériques, constituent simultanément une ressource mobilisable par des individus ou des groupes afin d'acquérir ou de maintenir des positions au sein de l'organisation ${ }^{59}$. Cela n'apparaît pas tant dans les discours de ceux qui mettent en place de telles initiatives, que dans les propos de ceux qui ne sont pas à l'origine de celles-ci et voient d'autres adhérents obtenir des positions au sein du parti, non sans une pointe de regret, à l'instar de ce socialiste :

«Prends, quand je regarde sur Paris, une partie des gens, des jeunes de mon âge qui... ont des responsabilités, qui vont au congrès fédéral ou des trucs, on s'en fout... Bah c'est des gens qui sont, en grande partie, qui sont soit passés par la campagne, soit par... avec François Hollande. 
C'est des gens qui ont été, beaucoup, beaucoup présents sur Internet. Les gens présents sur Internet là, ça joue quoi. Ils acquièrent une... une image, un réseau, une visibilité... extraordinaire, moi je trouve, par rapport à ce qui se passe (rire), ce qui se joue sur Internet. $»^{60}$

Une deuxième manière de faire consiste à créer des supports de communication plus à distance de l'organisation et de façon plus individuelle. Comme l'indique une enquêtée concernant sa page Facebook, il y est possible de faire «sa propre campagne ». Tout en intervenant sur une page de soutien à Nicolas Sarkozy, elle a transformé sa propre page Facebook en page publique :

« J'ai ma propre page Facebook. Alors... qui est partagée en différentes parties : la partie famille, la partie amis. Mais la ... ma propre page est une page publique sur laquelle, j'ai fait ma propre campagne, où j'ai mis tous les articles qui nous concernaient, tout ce qui n'allait pas avec les partis politiques. Et je continue à l'heure actuelle, etj'ai, je sais pas, peut-être huit cent abonnés ${ }^{61}$

Parfois, il s'agit de tenir sur ces pages des propos qui ne peuvent pas être tenus au nom du candidat ou de l'organisation officielle, comme dans le cas de pages qui tournent en dérision ou attaquent d'autres candidats :

«Alors, j'avais un groupe mais c'était un groupe un peu plus, un peu léger. Ça s'appelait «les galéjades $^{62}$ de Flanby». Ah ouais, parce qu'on l'a appelé Flanby pendant la campagne...C'était, son, son grand surnom. C'était pas un manque de respect, c'était voilà....C'était pour jouer le jeu de la campagne. Et on essayait de recenser tous les mensonges qu'il disait pendant la campagne, et tout ce qu'il voulait faire et ce qui était infaisable... $\nu^{63}$

Ici, les actes participatifs sont destinés à soutenir le candidat ou le parti, mais l'expression de ce soutien se manifeste de façon nettement plus relâchée que ne l'autoriserait la communication institutionnelle. Ces modes informels de contribuer à la campagne constituent en même temps une nouvelle façon de s'inscrire dans l'élan militant et de l'organiser et parfois d'atteindre des publics sympathisants peu réceptifs à l'égard de la communication partisane : la communauté créée autour du groupe Facebook ou du compte Twitter ne recoupe pas totalement celle de l'organisation politique et ne tire pas sa légitimité de celle-ci. Ainsi, l'enquêté créateur des « galéjades de Flanby » a aussi, au lendemain de l'élection présidentielle, créé un groupe Facebook de "Résistance française », consécutivement à l'élection de François Hollande car il trouvait « injuste» l'éviction de Nicolas Sarkozy. Cette initiative individuelle est, quelques mois plus tard, au moment de l'entretien, devenue une association regroupant environ 170 adhérents (selon l'enquêté), et un groupe " secret » sur Facebook. Parmi les membres de l'association, tous ne sont pas adhérents de l'UMP, mais tous reconnaissent le leadership de l'enquêté, confortant sa légitimité à organiser la mobilisation.

Une troisième manière de s'engager consiste à créer des supports tout à fait indépendants des organisations, en vue notamment de commenter l'actualité politique, plutôt que de se mobiliser ou de mobiliser activement pour un candidat spécifique. C'est le cas pour deux des personnes 
rencontrées, qui interviennent l'un sur un blog personnel de commentaire politique, sous pseudonyme ; l'autre sur un compte Twitter relié à un support satirique de gauche. Dans ce cas, la participation politique s'effectue en marge de toute organisation de campagne, et se joue sur un registre très distancié de la politique institutionnalisée. C'est par exemple le cas pour un enquêté ancien membre du PCF, exclu du parti au début des années 2000 mais qui se réclame toujours du communisme. Son activité numérique s'inscrit dans la survivance de cette mémoire, comme celle d'autres groupes à l'intérieur ou à l'extérieur du parti ${ }^{64}$. Il présente ainsi son activité de blogueur comme consistant à «porter un regard politique sur un événement», " apporter un point de vue qui prétend être un point de vue communiste», et mentionne notamment un article récent sur Paul Eluard. Pour lui, participer à la campagne a d'abord signifié ouvrir un blog, en 2007, «parce que...ça ne me plaisait pas comment la campagne s'engageait, j'avais des choses à dire.» Cinq and plus tard, il blogue toujours et sa participation à la campagne consiste essentiellement à diffuser ses points de vue. Il observe avec satisfaction une augmentation de l'audience de ses textes, même s'il ne se fait pas d'illusion quant à leurs effets :

«Elle avait commencé avant la campagne, c'està-dire pendant la pré-campagne, une diffusion qui a augmenté, qui m'a surpris, hein, très honnêtement. Au début, quand j'commençais à diffuser, si j'avais, allez... une trentaine de... une trentaine de lecteurs en moyenne par jour j'étais tout à fait satisfait. Là, quand j'suis... passé la campagne maintenant, quand j'suis à moins de 300, j'commence à m'dire, $y$ a des problèmes et je ... je tourne sensiblement, alors, en visiteurs en moyenne 400-500 jour... par jour dans la période, j'étais monté dans la partie la plus pointue de la campagne électorale, c'est-à-dire avant le premier tour, j'étais monté jusqu'à 600700. Alors ça relativise y compris l'importance du blog. Il est... c'est... les moteurs de recherche disent qu'il est assez conséquent, faut pas non plus se voiler la face, on fera pas la révolution avec ça. " ${ }^{65}$

Un autre enquêté dispose quant à lui de deux comptes Twitter - l'un individuel (sous pseudonyme), l'autre relié au site satirique auquel il contribue épisodiquement -, à partir desquels il tweete régulièrement. Dans l'entretien, il exprime un mélange de pitié et d'admiration pour le militantisme, qui lui est, dit-il étranger. Sa participation à la campagne se fait exclusivement sur le registre de l'humour, et il ne considère pas vraiment Twitter comme un espace de construction de l'action politique, plutôt comme un espace où peut s'exprimer librement toute forme de conflictualité ${ }^{66}$ :

«Pour moi c'est plus... c'est de la vanne de comptoir, un petit peu plus haut niveau quoi, mais, mais je suis pas sûr qu'on puisse... construire quelque chose dans un but politique. Par contre je pense que ça a une fonction, ça a une vocation exutoire qui pour moi est manifeste c'est-à-dire que... la prise de chou, de bec, avec un opposant... je pense, a aussi des vertus pour moi, à mon avis quasi-thérapeutique de plein de gens. $\gg{ }^{67}$

On retrouve un discours assez proche chez un autre enquêté, doctorant en sociologie, dont la position de spectateur, à distance du militantisme 
classique, est tout à fait assumée. Il ne participe à des discussions qu'à partir de son compte personnel Facebook.

« - Oui ? Pour participez à des discussions, comment ça se, ça prend forme?

- Bah, en bon étudiant socio, pour critiquer un peu... Sur Facebook, c'est de l'amusement, critiquer tout en charriant.... Eux, ils sont vraiment militants... ils ont été à toutes les manifs, etc... Moi, je ne milite (pas). Je charrie, tout en sachant qu'on a une communauté de vue quoi. $»^{68}$

Comme le montre la littérature consacrée aux usages de Twitter en campagne électorale, synthétisée par A. Jungherr, les commentaires sur la « course de chevaux » entre candidats sont prééminents sur ce réseau socionumérique, et l'humour, l'ironie, la satire y sont des registres d'intervention extrêmement répandus ${ }^{69}$. C'est pourquoi le même auteur préfère définir Twitter comme « un environnement communicationnel pour des affirmations phatiques, en réaction aux événements politiques, plutôt que comme un espace délibératif pour l'échange et le débat d'arguments politiques $\gg{ }^{70}$. Dans ce cadre, la participation à la campagne ne se fait plus sur le mode de l'engagement, même personnel, pour un candidat, mais sur le mode du commentaire politique.

Ainsi, la participation à la campagne revêt des configurations variées, qui diffèrent selon le rapport des enquêtés à l'organisation politique, au militantisme et à leur appétence pour tel ou tel type d'espace ou d'outil. Ils s'approprient les actes participatifs possibles, et la part de créativité qu'ils recèlent, sans nécessairement se conformer aux propositions des organisations mais bien plutôt relativement à leurs propres trajectoires et centres d'intérêts.

\section{Engager son réseau}

La participation à la campagne suppose presque toujours l'implication d'un réseau de relations, à des degrés divers : de l'exposition de soi à la sollicitation directe, l'internaute met en jeu et en scène ses attachements partisans comme son identité numérique entendue comme «la collection des traces (écrits, contenus audio ou vidéos, messages sur des forums, identifiants de connexion, etc.) que nous laissons derrière nous, consciemment ou inconsciemment, au fil de nos navigations sur le réseau et le reflet de cet ensemble de traces, tel qu'il apparaît "remixé " par les moteurs de recherche. ${ }^{71} \mathrm{Cet}$ engagement a un coût personnel, variable mais non négligeable pour certaines des personnes interrogées. Lorsqu'elle ne se fait pas au nom de l'organisation politique, la prise de parole est dès lors le plus souvent sous pseudonyme, la plupart des enquêtés cherchent ainsi-ils n'y parviennent pas toujours - à cloisonner les activités relatives à la campagne des autres activités ou relations associatives, amicales ou professionnelles. Comme le dit un enquêté blogueur :

« Disons qu'ça n'a pas forcément besoin d'apparaître dans le... dans l'enquête mais pour information, pour que vous compreniez, j'suis secrétaire général de (nom d'une association nationale). Et la diversité des gens qui... qui interviennent, qui agissent, qui s'y ... qui s'y consacrent ne correspond pas au profil personnel que j'ai ... J'ai pas envie de les embarquer 
derrière cette affaire-là. C'est mon propos, c'est pas l'leur. D'où le pseudo alors ...C'est un secret de polichinelle, hein, nombre d'entre eux sont au courant mais... C'est une question d'honnêteté vis-à-vis d'eux. $»^{72}$

Parmi les enquêtés, certains s'imposent des normes comportementales constitutives d'une « netiquette » qui définit les contours de ce qui est dicible ou non, comme de ce qui est visible ou non. La part prise à la campagne met en effet en question l'identité numérique du participant et l'activité en ligne peut alors se trouver limitée, sa teneur faisant l'objet d'une auto-négociation perpétuelle plaçant l'internaute dans une zone de « clair-obscur » identifiée par Dominique Cardon et propre aux réseaux socionumériques, particulièrement Facebook ${ }^{73}$.

Une des enquêtées explique comment elle entend protéger sa réputation, ainsi que celle des membres de sa famille, en s'abstenant de commenter et en transmettant simplement des articles de presse dont elle prend soin d'indiquer la source :

« Et même si j'ai pas commenté parce que en général, j'essaie, alors, j'essaie de ne pas trop m'impliquer. On connaît mes idées, mais j'essaie de ne pas trop m 'impliquer. Moi, je fais attention à la sécurité, et puis j'ai une fille qui est avocate, un gendre qui est avocat, donc je fais extrêmement attention de ne pas nuire, ni aux uns, ni aux autres, c'est une question quand même de, de déontologie, faut faire très attention. Donc moi quand je copie un article sur un magazine de presse... le petit commentaire que je pourrai mettre, je copie un extrait, et je mets à côté "ex- trait" $^{\prime \prime} \gg^{74}$

Si les réseaux socionumériques constituent de fait des espaces de dévoilement de soi avec une plus ou moins grande variabilité, le simple affichage des affinités partisanes suscite des réactions que l'enquêté anticipe parfois avec appréhension. Par exemple, pour cette jeune femme :

«Facebook, ça reste très pour le coup ... orienté quoi, enfin... Déjà, moi personnellement, avant de mettre «j'aime Jean-Luc Mélenchon»j'ai pesé mon truc, parce que je me suis dit «merde, mes copains, ils vont se foutre de ma gueule quoi!» (rire) $\gg^{75}$

En affichant de la sorte leurs convictions, certains enquêtés prennent le risque de se mettre à dos une partie du réseau de sociabilité qu'ils se sont constitués au fil du temps :

« Oui, d'ailleurs, je me suis inscrite, j'ai cliqué, donc, sur ma photo Facebook j'avais le badge et je me suis fait traiter de plein de noms d'oiseaux (rire) par des gens que je connais, mais j'ai répondu en disant que moi je respecte les convictions des autres. $»^{76}$

Sans que les internautes aient toujours conscience de la possibilité de paramétrer, dans une certaine mesure, le degré de publicité de leurs propos sur Facebook, les personnes que nous avons rencontrées peuvent faire penser aux enquêtés de Nina Eliasoph qui montre que les citoyens expriment davantage de préoccupations politiques dans les conversations les plus privées que dans les arènes qu'ils considèrent comme publiques ${ }^{77}$. De ce point de vue, Facebook apparaît comme 
un espace bien trop public pour y afficher sans hésitation des préférences partisanes, ainsi que le déclare l'un des enquêtés :

« Aujourd'hui, mon Facebook n'est plus qu'un Facebook politique et journalistique. Alors qu'avant la campagne, il ne l'était pas du tout! Il était pas grand-chose d'ailleurs en l'occurrence même si j'y étais depuis très longtemps mais comme je n'ai jamais rien mis de personnel. De ma vie privée... évidemment de mettre ses engagements c'est beaucoup plus...(...) privé que de mettre (...) ses, ses photos. Les vacances à la plage c'est pas dans un isoloir alors que le vote l'est. $\rangle^{78}$

La méfiance à l'égard de ces espaces est d'autant plus prononcée qu'elle s'accompagne d'une réflexion quant au statut des propos qui y circulent et qui peuvent être sujets à un fact checking quasipermanent, en particulier sur Twitter. S'exprimer sur Twitter revient alors à s'inscrire dans des conversations potentiellement illimitées.

«Avec Twitter vous pouvez très bien, un mec peut vous sortir je sais pas, le coût du travail machin (...), vous avez la possibilité en une minute d'aller vérifier si ce que présente, enfin si l'affirmation qui est balancée est vraie, est fausse ou tout du moins est modulable. (...) Donc en fait et ça je pense, je sais pas si ça peut vraiment changer la politique mais... les assertions et les coups de menton en disant «telle chose coûte tant et si on fait comme ça il y aura un indicateur de tant de points en plus et cetera et cetera»,... J'ai posé ce simple souhait mais j'ai le sentiment que ça commence un peu à s'étioler parce que si vous dites ça, dans un débat sur Twitter y a 50 mecs qui vont sauter sur le truc et qui vont... qui iront balancer un lien qui va soit vous contredire, soit au contraire essayer d'enfumer, soit au contraire valider des propos qui sont avancés. ${ }^{79}$

Les tensions entre ce qui relève du politique, du public et du privé sont également observables lorsqu'il s'agit d'appréhender non plus le partage d'informations ou les divers modes d'affichage des convictions mais les manières dont les internautes vont tenter d'entrer en contact les uns avec les autres. Pour certains enquêtés, les réseaux socionumériques constituent des modes de contact moins intrusifs comparés aux formes antérieures de communication numérique telles que le courrier électronique.

«Voilà j'ai, j'ai tweeté, retweeté, partagé énormément de choses sur Facebook etc. Mais il y a un truc qui est amusant. C'est qu'à un moment le mail est arrivé chez les gens comme une manière... de... de s'adresser à eux sans... forcer leur intimité. Au début c'est ce qu'on se disait, ce qui était bien, vous voulez rencontrer quelqu'un, ou le contacter, vous le contactez par mail. Et puis l'évolution des outils numériques ayant fait qu'effectivement cette fonction a été justement remplacée par Facebook, par Twitter, par les réseaux sociaux et ce qui fait qu'aujourd'hui que le mail devient quelque chose de beaucoup plus intrusif personnellement. Et au bout d'un moment effectivement, moi sur les gens que j'ai dans mes réseaux par mail... je ne me sentais pas forcément le droit de rentrer par la fenêtre politique, par là. Alors que je me sentais beaucoup plus le droit de leur envoyer un tweet ou un machin ou dans mes listes de diffusion, voilà... $»^{80}$ 


\section{Conclusion}

Notre travail rejoint des observations effectuées à partir d'autres objets, comme par exemple le porte-à-porte à propos duquel Rémi Lefebvre (2016) montre que même les personnes les plus engagées dans les campagnes électorales, entretiennent une distance certaine à l'égard des choix, du marketing électoral et des stratégies élaborées par les équipes de campagne. De fait, la figure du « bon » militant qui mobiliserait son réseau de connaissances interpersonnelles auquel il diffuserait programmes et communiqués de presse et qu'il convierait à des sessions de live-tweet ou à des meetings en direct live se heurte à celle du citoyen rétif à l'embrigadement partisan et sensible à la protection de la vie privée lorsqu'il s'agit de diffuser ou manifester ses convictions politiques.

Par ailleurs, l'enquête réalisée auprès de personnes impliquées dans la campagne permet de saisir, au-delà de l'homogénéité sociographique indéniable de cette population, la diversité des modes d'appropriations des dispositifs numériques. Ce que l'on entend par «participer à la campagne numérique » renvoie à tout un ensemble de pratiques et de significations, du commentaire sous pseudonyme à la création de pages partisanes, en passant par l'animation des pages ou des comptes personnels. La participation politique en ligne ne consiste donc pas seulement à la transposition de modalités de participation politique hors ligne : elle recouvre des « actes participatifs » plus ou moins tournés ou éloignés des organisations, et mettant plus ou moins en jeu le réseau personnel de l'internaute. Il se joue donc, autour des dispositifs sociotechniques de communication, des recompositions dans les possibilités de prendre la parole et de se mettre en scène pour la fraction des citoyens les plus engagés, les plus politisés et disposant des compétences informatiques pour le faire. Ces possibilités de prendre la parole et de se mettre en scène pourraient conduire à réviser ce que participer veut dire : pour les internautes engagés, il s'agit d'abord de créer du contenu et de s'exprimer. Ces possibilités ne sont pas, on le sait, socialement distribuées de manière égale. Mais, politiquement, elles concernent l'ensemble du spectre politique et appellent donc une réflexion sur les modalités de l'engagement et le rapport à l'organisation médiatisé par les technologies numériques. 
$\mathrm{N} \cdot \mathrm{O} \cdot \mathrm{T} \cdot \mathrm{E} \cdot \mathrm{S}$

1. F. Greffet, "New techniques, new mobilizations? French Parties in the Web 2.0 era", in Paul G.Nixon, Rajash Nawal et Dan Mercea (eds), Politics and the Internet in Comparative Context, Routlege, 2013, p. $59-74$.

2. Voir notamment le numéro spécial coordonné par D. G. Lilleker, K. Koc-Michalska, R. Negrine, R. Gibson, T. Vedel et S. Strudel, "Social media campaigning in Europe. Mapping the terrain", Journal of Information Technology \& Politics, vol. 14, n 4 , 2017, p. 293 et suivantes.

3. A. Theviot, "Les data : nouveau trésor des partis politiques ? Croyances, constitutions et usages comparés des données numériques au Parti Socialiste et à l'Union pour un Mouvement Populaire ", Politiques de communication, vol. 6, n . 1, 2016, p. 137-166 ; D. Karpf, Analytic Activism. Digital Listening and the New Political Strategy, Oxford University Press, 2017.

4. R. Lefebvre et E. Treille (dir.), Les primaires ouvertes en France, Rennes, Presses universitaires de Rennes, 2016.

5. Respectivement en-marche.fr, lancé en avril 2016; et jlm2017.fr, lancé en février 2016.

6. Pour la campagne de 2012, voir S. Gadras, F. Greffet, «La présence web des candidats en 2012. Espaces officiels et espaces non-officiels de campagne » in Jacques Gerstlé et Raul Magni-Berton (dir), 2012, la campagne présidentielle. Médias, électeurs et candidats dans la campagne de 2012. Paris, éditions Pepper, 2014, 73 87 ; C. Mabi et A. Théviot, «La rénovation par le web ? Dispositifs numériques et évolution du militantisme au PS », Participations, vol. 1, n8, 2014, p. 97-126. 7. Les statistiques sont issues de la base de données Eurostats (variable « utilisation d'Internet par les particuliers », disponible en ligne $: \underline{\text { http://ec.europa.eu/ }}$ eurostat/fr/home) et du document ARCEP « Baromètre du numérique 2017 », également disponible en ligne : https://www.arcep.fr/uploads/tx_gspublication/ barometre_du_numerique-2017-271117.pdf. Sources consultées le 12 juin 2018.

8. S. Wojcik, "Prendre la démocratie électronique au sérieux. De quelques enjeux et controverses sur la participation politique en ligne », in E. Forey et C. Greslot (dir.), Internet, machines à voter, démocratie, L'Harmattan, 2011, p. 111-141.

9. K. Koc Michalska, Darren G. Lilleker et Th. Vedel, "Civic political engagement and social change in the new digital age”, New Media \& Society, vol. 18(9), 2016, p. 1807-1816.

10. J. Boyadjian, Analyser les opinions politiques sur Twitter, enjeux théoriques et défis méthodologiques, Paris, Dalloz, 2016.

11. M. J. Jensen et al., "Introduction", in M. J. Jensen, L. Jorba, E. Anduiza (eds), Digital media and political engagement worldwide, Cambridge University Press, 2012, p. 1-15.

12. E. Morozov, "The brave new world of slacktivism”, 2009, Foreign Policy, publié en ligne : http:// foreignpolicy.com/2009/05/19/the-brave-new-worldof-slacktivism/, page consultée le 22 juin 2018). Et, du même auteur The Net Delusion. The Dark Side of Internet Freedom, New York, PublicAffairs, 2011.

13. Le projet était intitulé "enpolitique.com : stratégies, contenus et perceptions des usages politiques du web en période électorale. Le cas des campagnes électorales présidentielle française et législative québécoise ». Il a été financé en France par l'Agence Nationale de la Recherche (Programme de collaboration France-Québec en SHS) et au Québec par le Fonds de Recherche Québec - Société et Culture. La responsabilité scientifique du projet incombait à Fabienne Greffet (Université de Lorraine) 
et Thierry Giasson (Université Laval). Participaient également à ce projet en France : Gersende Blanchard (Université Lille 3), Simon Gadras (Université Lyon 2), Stéphanie Wojcik (Université Paris-Est Créteil) et au Québec : Frédérick Bastien (Université de Montréal) et Mireille Lalancette (Université du Québec à Trois Rivières).

14. Cette approche est notamment mobilisée dans le cadre d'enquêtes internationales comme on peut le voir dans le module " citoyenneté » de l'ISSP (International Social Survey Programme) de 2014 teste plusieurs modes de participation politique, comptabilisés de la même façon qu'ils soient réalisés en ligne ou par le biais d'une interaction physique. Cf. « Master questionnaire » du module « citizenship II » de 2014, http://issp.org/data-download/by-topic/, consulté le 22 juin 2018.

15. W. Lance Bennett \& Alexandra Segerberg, "The logic of connective action", Information, Communication \& Society, 15:5, 2012, p. 739-768,

16. B. Bimber, "Digital media and citizenship", in H. A. Semetko and M. Scammell (eds), The Sage Handbook of Political Communication, Sage Publications, 2012, p. 115-126.

17. P. N. Howard, New Media Campaigns and the Managed Citizen. Cambridge, UK: Cambridge University Press, 2006.

18. La forme passive est une mesure combinée de : visiter le site web d'une municipalité/gouvernement/ administration publique et n'importe quel site web avec un contenu politique. La forme active est un ensemble d'activités nouvelles (propres au web) et d'équivalents de ce qui se fait hors ligne (c'est-à-dire réagir en ligne à un message ou un article; signer une e-pétition et participer à des sondages en ligne). T. P. Bakker, C. H. de Vreese, "Good News for the Future? Young People, Internet Use, and Political Participation", Communication Research, 2011, 38
(4), p. 451-470.

19. M. Cantijoch, R. Gibson, D. Cutts, "Engagement in the online campaign in the United States and France. A comparative analysis", ECPR General Conference, Bordeaux, France, September 4-7, 2013. 20. Cette catégorisation est davantage justifiée et explicitée dans F. Greffet, S. Wojcik, G. Blanchard, «S'engager dans la campagne présidentielle. Les formes multiples de la participation politique en ligne ", Politiques de communication, ${ }^{\circ} 3$, p. 25-58, 2014, ainsi que dans F. Greffet et S. Wojcik, "Exploring Online Participation: The Case of the French Presidential Election Campaign, 2012"; Comunicazione Politica, 2017/vol. 1, p. 3-30, 2017.

21. L. Monnoyer-Smith, « La participation en ligne, révélateur d'une évolution des pratiques politiques? », Participations, $\mathrm{n}^{\circ}$ 1, 2011, p. 156-185.

22. H. Jenkins, M. Ito, d. boyd, Participatory Culture in a Networked Era, Polity Press, 2016.

23. Y. Theocharis, "The conceptualization of digitally networked participation”, Social Media + Society, July-December 2015, p. 6 (notre traduction).

24. F. Greffet, S. Wojcik et G. Blanchard, op. cit. ; F. Greffet et S. Wojcik, op. cit.

25. Les recherches tendent à se focaliser sur Twitter, au détriment d'autres dispositifs.

26. S. Gadras et F. Greffet, "Guest Editorial: towards a comprehensive definition of online political participation", International Journal of Electronic Governance, vol. $6, \mathrm{n}^{\circ} 4,2013$, p. 263.

27. D. Kreiss, Taking Our Country Back. The Crafting of Networked Politics from Howard Dean to Barack Obama, Oxford University Press, 2012 et Prototype Politics, Technology-Intensive Campaigning and the Data of Democracy, Oxford and New York, Oxford University Press, 2016.

28. A. Chadwick, The Hybrid Media System. Politics and Power, Oxford University Press, 2013. 
29. A. Théviot, «Les Data: nouveau trésor des partis politiques? Croyances, constitutions et usages comparés des données numériques au parti socialiste et à l'Union pour un mouvement populaire ", Politiques de Communication 1(6), 2016, 137-166. De la même manière, les candidats à l'élection de 2017 n'ont pas hésité à recourir aux services de divers agences et prestataires - tels que Liegey Muller Pons, NationBuilder ou Digitalebox - qui emploient des algorithmes pour analyser une diversité de données en vue d'adapter les stratégies de communication et de mobilisation tout au long de la campagne.

30. A. Coutant et T. Stenger, « Processus identitaire et ordre de l'interaction sur les réseaux socionumériques », Les Enjeux de l'information et de la communication, $\mathrm{n}^{\circ} 1,2010$, p. 63.

31. J. Jouët, R. Rieffel (dir.), S'informer à l'ère numérique, Rennes, Presses universitaires de Rennes, 2013.

32. Pour les États-Unis, voir les enquêtes du Pew Research Center, "Internet now major source of campaign news", 31 October 2008, http://pewresearch. org/pubs/1017/Internet-now-major-source-of-campaign-news ; Pew Research Center, «Election 2016: Campaigns as a Direct Source of News», 18 July 2016, http://www.journalism.org/2016/07/18/election2016-campaigns-as-a-direct-source-of-news/, source consultée le 22 juin 2018. En France, T. Vedel note, d'après les enquêtes électorales du CEVIPOF que pour l'élection présidentielle de 2012, 35 \% des Français déclaraient qu'Internet constituait leur première ou seconde source d'information politique, contre $16 \%$ en 2007 », in «L'internet et la démocratie : une liaison difficile », in Perrineau P., Rouban L. (dir.), La démocratie de l'entre-soi, Presses de Sciences Po (P.F.N.S.P.), 2017, p. 73-88.

33. G. Le Saulnier, « La lecture de la presse en ligne. L'appropriation des contenus d'actualité au défi de la technique », in J. Jouët, R. Rieffel (dir.), S'informer à l'ère numérique, Rennes, Presses universitaires de Rennes, 2013, p. 63.

34. Entretien 4, M., 66 ans, militaire retraitée, adhérente UMP depuis mai 2012 (Metz).

35. V. Le Hay, T. Vedel, F. Chanvril, « Usages des médias et politique : une écologie des pratiques informationnelles ", Réseaux, n¹70, 2011, p. 45-73.

36. Entretien 17, S., 44 ans, informaticien, de gauche, n'adhère à aucun parti (Paris).

37. Entretien 20, J., 49 ans, chef d'entreprise (développement informatique), adhérent PS depuis plusieurs années (Lyon).

38. L'engouement des internautes pour les formats vidéo se confirme durant l'élection de 2017 avec une importante utilisation de YouTube par les candidats, qu'il s'agisse de créer sa propre chaîne (J.-L. Mélenchon) ou de produire des émissions de type plus classique (E. Macron, F. Fillon).

39. Entretien 1, A., retraitée 63 ans, conseillère commerciale polyvalente banque, sympathisante UMP (Paris).

40. Entretien 6, A., 37 ans, commercial au chômage, sympathisant UMP durant la campagne, a adhéré en juin 2012 (Metz).

41. Entretien 12, J-L, 47 ans, consultant indépendant (carrière totalement dans le privé), PS.

42. Entretien 17, S., 44 ans, informaticien, de gauche, n'adhère à aucun parti (Paris).

43. Entretien 10, F., 27 ans, attachée territoriale, sympathisante du Front de Gauche (Lille).

44. Entretien 13, G., 60 ans, cheminot retraité, exadhérent PCF, communiste (Paris).

45. Voir par exemple T. Small, "Equal Access, Unequal Success - Major and Minor Canadian Parties on the Net”, Party Politics, vol. 14, n 1, 2008, 51-70 ; J. Stromer-Galley, "On-line interaction and why candidates avoid it”, Journal of Communication, Volume 
50, Issue 4, December, 2000, 111-132; F. Bastien et F. Greffet, « Les campagnes électorales sur Internet : une comparaison entre France et Québec », Hermès, vol. 54, no. 2, 2009, p. 211-219.

46. N. A. Jackson, D. G. Lilleker, "Building an architecture of participation? Political parties and web 2.0 in Britain", Journal of Information Technology and Politics, vol. 6, issue 3-4, 2009, p. 232-250.

47. D. Cardon, G. Fouetillou, C. Lerondeau, C. Prieur, «Esquisse de géographie de la blogosphère politique (2007-2009) », in F. Greffet (dir.), Continuerlalutte. com, Les partis politiques sur le web, Paris, Presses de Sciences Po, 2011, p. 93.

48. Entretien 19, R., 28 ans, en recherche d'emploi (juriste dans l'environnement), adhérent Modem depuis 2007 (Lille).

49. S.Roginsky et B. De Cock, « Faire campagne sur Twitter. Modalités d'énonciation et mises en récit des candidats à l'élection européenne », Les Cahiers $d u$ numérique, vol. 11, n4, 2015, p. 119-144.

50. Entretien 5, V., 28 ans, chargée de projet dans une association de solidarité internationale, adhérente à aucun parti.

51. Entretien 4, M., 66 ans, militaire retraitée, adhérente UMP depuis 2012.

52. Entretien 1, A., retraitée 63 ans, conseillère commerciale polyvalente, banque, sympathisante UMP (Paris).

53. Entretien 7. P.-Y., 51 ans, directeur de Segpa (section d'enseignement général et professionnel adapté), MODEM (Lille).

54. Entretien 18, O., 20 ans, étudiant en droit, adhérent des Jeunes Populaires depuis 2010 (Metz).

55. Entretien 14, N., 25 ans, stagiaire dans un cabinet de communication et d'affaires publiques, adhérent PS depuis 2011 (Paris).

56. R. Lefebvre, « La modernisation du porte-à-porte au Parti socialiste. Réinvention d'un répertoire de campagne et inerties militantes », Politix, $n^{\circ} 113$, p. 107.

57. Entretien 2, F., 24 ans, étudiant en médecine, adhérent au PCF depuis 2011 (Nancy).

58. Entretien 18, O., 20 ans, étudiant en droit, adhérent des Jeunes Populaires depuis 2010 (Metz).

59. L. Bargel et A.-S. Petitfils, « Appropriations d'Internet et trajectoires militantes «dans» et «en-dehors» de l'UMP », in F. Greffet (dir.), Continuerlalutte. com, Les partis politiques sur le web, Paris, Presses de Sciences Po, 2011, 187-199 ; A. Théviot, Mobiliser et militer sur Internet. Reconfiguration des organisations partisanes et du militantisme au Parti Socialiste et à l'Union pour un Mouvement Populaire, thèse de doctorat en science politique, Université de Bordeaux, soutenue le 10 octobre 2014.

60. Entretien 14, N., 25 ans, stagiaire dans un cabinet de communication et d'affaires publiques, adhérent PS depuis 2011 (Paris).

61. Entretien 1, A., 63 ans, conseillère commerciale polyvalente, banque (retraitée), sympathisante UMP (Paris).

62. Le nom du groupe a été modifié pour préserver l'anonymat de l'enquêté.

63. Entretien 6, A., 37 ans, commercial au chômage, sympathisant UMP durant la campagne, a adhéré en juin 2012 (Metz).

64. D. Andolfatto, "Explorer la planète rouge ", in F. Greffet (dir.), Continuerlalutte.com, Les partis politiques sur le web, Paris, Presses de Sciences Po, 2011, p. 153-161.

65. Entretien 13, G., 60 ans, cheminot retraité, exadhérent PCF, communiste (Paris).

66. De ce point de vue, la campagne de 2017 montrera un usage véritablement subversif de Twitter de la part des internautes qui n'hésiteront pas à produire et véhiculer des informations peu vérifiées à propos des différents candidats et à les critiquer durement : A. 
Mercier, « Contestation et manipulations électorales en 140 signes ", in Perrineau P. (dir.), Le vote disruptif. Les élections présidentielle et législatives de 2017, Presses de Sciences Po, 2017, p. 115-128.

67. Entretien 17, S., 44 ans, informaticien, de gauche, n'adhère à aucun parti (Paris).

68. Entretien 16 : J.-P., 32 ans, doctorant en sociologie, n'adhère à aucun parti (Paris).

69. A. Jungherr, "Twitter use in election campaigns: A systematic literature review", Journal of Information Technology \& Politics, 2016, 13 :1, p. 72-91.

70. Traduction libre de : "it seems more plausible to conceptualize Twitter as a communication environment for phatic statements in reaction to political events than as a deliberative space for the exchange and debate of political arguments." A. Jungherr, ibid., p. 78.

71. O. Ertzscheid, Qu'est-ce que l'identité numérique? Enjeux, outils, méthodologies, OpenEdition Press, 2013, p. 15.

72. Entretien 13, G., 60 ans, cheminot retraité, exadhérent PCF, communiste (Paris).

73. D. Cardon, « Le design de la visibilité » Un essai de cartographie du web 2.0», Réseaux, 2008/6, n 152. 74. Entretien 4, M., 66 ans, retraitée de l'armée où elle était technicienne informatique, adhérente UMP depuis 2012.

75. Entretien 10, F., 27 ans, attachée territoriale, sympathisante du Front de Gauche (Lille).

76. Entretien 1, A., 63 ans, conseillère commerciale polyvalente banque (retraitée), sympathisante UMP (Paris).

77. N. Eliasoph, L'évitement du politique. Comment les Américains produisent l'apathie dans la vie quotidienne, Économica, coll. «Études Sociologiques », 2010.

78. Entretien 15, B., 44 ans, compositeur pour l'audiovisuel et journaliste indépendant, animateur d'un think tank de gauche, sympathisant socialiste (Paris).

79. Entretien 17, S., 44 ans, informaticien, de gauche, n'adhère à aucun parti (Paris).

80. Entretien 15, B., 44 ans, compositeur pour l'audiovisuel et journaliste indépendant, animateur d'un think tank de gauche, sympathisant socialiste (Paris). 
$R \cdot E ́ \cdot S \cdot U \cdot M \cdot E ́$

À l'aide d'une typologie d'actes participatifs en ligne, le texte explore les formes numériques d'engagement durant la campagne présidentielle française de 2012 à partir d'entretiens auprès d'internautes. Deux spécificités paradoxales de ces formes d'engagement sont particulièrement analysées. D'une part, la créativité dont font preuve les internautes dans l'appropriation des dispositifs numériques se heurte à la volonté de «management» de leur activisme par les équipes de campagne. D'autre part, dans les campagnes en ligne, les internautes mettent en jeu et en scène leurs attachements partisans comme leurs identités numériques, notamment par l'exposition de leurs convictions ou la sollicitation directe via les réseaux socionumériques. Cela peut conduire à mettre en tension la participation numérique avec le risque réputationnel qu'elle représente. La figure du citoyen engagé apparaît ainsi rétive à l'embrigadement partisan et sensible à la protection de la vie privée lorsqu'il s'agit de diffuser ou manifester ses convictions politiques.

\section{Abstract \\ Renewing political participation. The invention of individualized and critical modes of digital engage- ment in the election campaigns.}

Using a categorization of online political participation acts as a basis, this paper investigates digital forms of activism during the 2012 French presidential campaign through interviews with Internet users. Two paradoxical trends are underlined. On the one hand, the users that engage online in order to support a candidate or a party sometimes face the «management» of activism operated by campaign teams, which might undermine their own creativity. On the other hand, Internet users display their beliefs or directly ask for support to the people they are connected with, especially on social networks. In this case, Internet users stake and stage their political commitments and digital identity, which can lead to reputational risks. Thus, committed citizens are reluctant to partisan propaganda and sensitive to the protection of their own privacy, when it comes to displaying their political convictions. 
